\title{
Aspectos relevantes del diseño, gestión e impactos del programa de Fomento Agrícola en el estado de Yucatán, 2002-2005
}

\author{
Miguel Ángel Magaña Magaña* \\ Carlos Enrique Leyva Morales**
}

\section{Resumen}

En este estudio se analiza el proceso de evaluación de políticas públicas en el subsector agrícola, específicamente el programa Alianza Contigo (antes Alianza para el Campo); además, se establece su situación, se determina su influencia en el impacto del Programa de Fomento Agrícola de Yucatán, del 2002 al 2005, y se observa su eficacia y eficiencia.

Se observó que la evaluación de políticas del Programa se efectúa por objetivos. Los principales resultados económicos y sociales, derivados de su operación, fueron la limitada capitalización (atomización del apoyo), tecnificación de las unidades de producción (mejora técnica y variedades tolerantes a VTC — virus de tristeza de los cítricos-). También se observó que hubo apoyo insuficiente a grupos de productores y a la población rural; asimismo, que se dio pocos incentivos para reducir la emigración a ciudades o para la generación de fuentes de empleo temporal.

De igual forma, con respecto al proceso de evaluación, se encontró que no es objetivo porque no cumple con el seguimiento; además, la metodología es limitada y no evalúa la eficiencia del propio Programa.

Palabras clave: subsector agrícola de Yucatán, evaluación de políticas públicas, Programa de Fomento Agrícola.

Fecha de recepción: 09/11/2007

Fecha de aceptación: 02/06/2008

\footnotetext{
*Profesor de tiempo completo en el Instituto Tecnológico de Conkal,

Correo electrónico: mmagana@itaconkal.edu.mx

**Profesor de tiempo completo en la Universidad Autónoma de Yucatán.

Correo electrónico: clmoral@tunku.uady.mx
} 
Miguel Ángel Magaña Magaña y Carlos Enrique Leyva Morales

\title{
Relevant aspects of the design, implementation and results of the Fomento Agrí- cola Program in the state of Yucatan, 2002-2005
}

\begin{abstract}
This study analyzes the public policy evaluation process in the agricultural subsector. Specifically the Alianza Contigo Program (formerly Alianza para el Campo). The aim is to establish its situation, determine its influence on the impact of the Yucatan 2002-2005 Fomento Agrícola Program (Programa de Fomento Agrícola), as well as to observe both its effectiveness and efficiency.

It was found that policy evaluation of the Program is by objectives. The main economic and social results derived from its operation were limited capitalization (atomized support), technification of agriculture production units (technical improvement and VTC -citrus sadness virus- tolerant strains). There was insufficient support to producers and rural population, few incentives to reduce migration into cities or for the generation of temporary employment sources.

Likewise, in regard to the evaluation process it was found as non objective since it does not follow up each case. Also the methodology is limited and it does not evaluate the Program's own efficiency.
\end{abstract}

Key words: Yucatan's agricultural subsector, public policy evaluation, Fomento Agrícola Program.

\section{Introducción}

T a evaluación de políticas públicas es una práctica de utilidad tanto para gestores y $\amalg$ responsables de las decisiones del sector gubernamental, ya que les brinda las herramientas para la mejora del diseño, gestión y ejecución de sus actuaciones, como a todos los interesados del impacto de los planes y programas públicos aplicados. Esta práctica está adquiriendo, en el mundo, una creciente importancia como herramienta debido a que genera información para una sociedad que demanda respuestas de la intervención pública, donde la eficacia, eficiencia y transparencia se convierten en las expresiones más inequívocas de la nueva intervención del Estado en la economía.

En México, la planificación adquirió un papel muy importante a partir de la década de los años cincuenta y en la actualidad la realizan tanto el gobierno federal, estatal, como el municipal, aunque en esta última la práctica de evaluación del proceso de elaboración, gestión y ejecución es limitada, sobre todo en los programas que se desarrollan en los estados. Por lo tanto, el seguimiento de planes y programas deben estar integrados con la gestión diaria de los centros directivos de instituciones o dependencias del gobierno debido a que en la administración pública juegan un 
Aspectos relevantes del diseño, gestión e impactos del programa

de Fomento Agrícola en el estado de Yucatán 2002-2005

papel fundamental los conocimientos y habilidades para la aplicación y desarrollo de técnicas evaluativas. En este proceso es importante avanzar en el perfeccionamiento de éstas, así como en su adaptación a los procedimientos, formas organizativas y culturales propias del sector público nacional.

En lo particular, la evaluación de los programas que se derivan de la política agrícola en el país ha buscado constituirse en el medio para mejorar el diseño e instrumentación, con miras a fomentar un desarrollo armónico de sus actividades productivas. En el ámbito institucional, y entre las varias instancias y programas de atención al subsector, sobresale el de Alianza para el Campo, del cual forma parte el de Fomento Agrícola (FA). Pero a pesar de que se han logrado avances con relación a estos dos aspectos de la política agrícola nacional se observan vacíos y retos pendientes que exigen revisar y valorar la orientación y enfoque tanto de la política actual como los instrumentos de intervención y evaluación.

Dado este contexto, esta investigación buscó contribuir al reconocimiento de la importancia de la evaluación de políticas públicas en el subsector agrícola, desde la fase de detección de problemas y necesidades hasta la de ejecución y conclusión del programa, trazándose como objetivo central el analizar el proceso de evaluación del Programa de Fomento Agrícola con el fin de diagnosticar su situación y determinar su influencia en los impactos al subsector agrícola del estado de Yucatán, así como observar su eficacia y eficiencia.

\section{Metodología}

El trabajo se basó en un diseño de comprobación de hipótesis del tipo no experimental longitudinal de tendencia. La información de interés se recopiló utilizando técnicas de investigación documental y la entrevista a informantes clave ${ }^{3}$. La guía utilizada en la entrevista se estructuró con preguntas respecto a los resultados económicos y sociales del Programa, a sus bases normativas y a la evaluación del diseño, gestión, seguimiento e impactos de mismo; los informantes se ubicaron en la Secretaría de Agricultura, Ganadería y Pesca (SAGARPA), Apoyos y Servicios a la Comercialización Agropecuaria (ASERCA) y en la Secretaría de Desarrollo Rural y Pesca (SDRyP) del gobierno del estado.

El análisis del proceso de evaluación del Programa de Fomento Agrícola se realizó con base en los principios teórico-metodológicos propuestos por Osuna y Marquez ${ }^{4}$

\footnotetext{
${ }^{3}$ R. Hernández, C. Fernández y P. Baptista, (1991), Metodología de la investigación, McGraw Hill, México.

${ }^{4}$ J. Osuna, y C. Márquez, (2000), Guía para la evaluación de políticas públicas, Instituto de Desarrollo Regional, España.
} 
que establecen que la evaluación de políticas públicas se debe realizar en todas sus etapas (diseño, gestión, seguimiento e impactos), analizando la pertinencia y coherencia de los problemas por resolver, objetivos e instrumentos planteados para tal efecto, buscando conocer hasta qué punto el programa aplicado está consiguiendo los objetivos establecidos y a qué costo (tiempo, recursos humanos, recursos materiales y monetarios). El primer caso se refiere a la evaluación de eficacia, mientras que al considerar los costos se habla de evaluación de la eficiencia ${ }^{5}$ (esta última busca deslindar los efectos del programa de otros externos para determinar en qué medida los cambios fueron debidos a la intervención). Lo anterior se complementó con la consideración de los aspectos técnicos de la evaluación de programas sociales propuestos por Cohen ${ }^{6}$ l la magnitud del gasto, el porcentaje del gasto efectivamente redistributivo y su impacto en la solución del problema. La información estadística analizada se centró en el comportamiento de las siguientes variables: ejercicio financiero, tipo y número de beneficiarios, volumen y valor de la producción agrícola, productividad, empleo e ingreso bruto de los productores agrícolas.

En general, las aproximaciones metodológicas consideradas en el estudio fueron:

1.- Evaluación centrada en objetivos. Utiliza como parámetro de referencia los objetivos de la política evaluada para efectuar mediciones comparadas entre lo pretendido inicialmente y lo realmente conseguido ${ }^{7}$.

2.- Modelos de aproximación clínica o analítica. Se emplean para explicar por qué un objetivo propuesto no ha sido alcanzado y por qué otros, no buscados en principio, sí lo han sido. Tratan de descubrir las diferencias entre la meta perseguida y la alcanzada efectivamente, considerando las condiciones en la que se ha ejecutado la política, intentando responder a cuestiones de tipo causa-efecto 8 .

3.- Modelo basado en la utilización de los resultados de la evaluación. Éste identifica la adaptación del proceso de evaluación a las necesidades del destinatario que encarga ésta en la aplicación de métodos cuantitativos para tal fin y en la implicación de distintos grupos de interés en el proceso evaluativo ${ }^{9}$.

4.- La metaevaluación. Se define como la evaluación de la evaluación de políticas y consiste en valorar sobre criterios, estándares e indicadores la calidad (validez,

\footnotetext{
${ }^{5}$ Teresa, García y Yolanda Calzado (1996), “Metodología de evaluación de la eficiencia en las entidades públicas”, Presupuesto y Gasto Público, No 18, pp. 189-202.

${ }^{6}$ Ernesto, Cohen (2001), "Los desafíos de la reforma del Estado en los programas sociales: tres estudios de caso", Santiago de Chile, CEPAL.

${ }^{7}$ A. S., Brik (1983), "Stakeholder-based evaluation”, New Directions for program evaluation, pp. 3-14.

${ }^{8}$ I., Meny y J. C. Thoening (1992), Las políticas públicas, Barcelona: Ariel Ciencia Política.

${ }^{9}$ J. S., Wholey (1983), Evaluation and effective public management, Boston: Littie Brown. 
Aspectos relevantes del diseño, gestión e impactos del programa

de Fomento Agrícola en el estado de Yucatán 2002-2005

utilidad y objetividad) de instituciones o programas ${ }^{10}$. Las estructuras teóricas más utilizadas en ella son los requerimientos de validez asociados con la práctica científica tradicional ${ }^{11}$, los estándares desarrollados por asociaciones profesionales ${ }^{12}$ y los procedimientos propuestos por autores independientes ${ }^{13}$.

En México, entre algunos de los estudios más recientes realizados sobre este tema, está el de Juan Trujillo, el cual arroja (basándose en la metodología de la OCDE) que los logros de las reformas en los noventas a las políticas agrícolas de Estados Unidos y la Unión Europea respecto a México han quedado por debajo de las expectativas esperadas, pero que ha habido un desplazamiento hacia una mayor desconexión de las políticas mexicanas respecto a las decisiones de producción y precios con detrimento hacia los productores agrícolas del país. Por su parte, Miriam Cardozo analiza los cambios introducidos en las políticas sociales por el neoliberalismo y particularmente su preocupación por la eficiencia, la cual se traduce en un marcado interés por evaluar los programas sociales en búsqueda de los mejores desempeños en términos de costo-beneficio; concluye que se ha tenido un avance significativo en la materia, pero que aún subsisten problemas importantes por resolver para que las evaluaciones sean metodológicamente confiables y útiles. Un tercer trabajo, el realizado por John Scott, señala que aunque el gasto público en subsidios agrícolas y el desarrollo rural en México es modesto en el contexto de la OCDE resulta de los más altos dentro de la región de América Latina y el Caribe en relación con el PIB agrícola y a otras demandas del gasto público y que los subsidios a la producción e insumos, como los mecanismos de apoyo vía precios del pasado, demostraron ser inefectivos para la transformación de la agricultura y extremadamente inequitativos.

\section{Resultados}

Programa Alianza para el Campo. Alianza para el Campo (APC), hoy Alianza Contigo (AC), surgió en 1996 como una respuesta de la Administración Federal para coadyuvar en la solución de los problemas que aquejaban al campo mexicano, cuyo énfasis se centró en la capitalización de las unidades de producción y en el desarrollo

\footnotetext{
${ }^{10}$ E. Rebolloso, et al. (2002), "Metaevaluation of a total quality management evaluation system”, Psychology in Spain, pp. 12-25.

${ }^{11}$ E. Rebolloso, et al. (2000), "El papel de la investigación cualitativa en la evaluación de los servicios universitarios”, Cuadernos IRC, pp. 65-82.

${ }^{12}$ JCSEE (1988), "The personnel evaluation standards: How to assess systems for evaluating educators", Newbury Park, CA: Sage; D.L. Stufflebeam, y A.J. Shrikfield (1987), "Evaluación sistemática: guía teórica y práctica”, Barcelona, Paidos.

${ }^{13}$ M. Scriven (1969), “An introduction to metaevaluation”, Educational Product Report, pp. 36-38; E. Chelimsky (1983), “The definition and measurement of evaluation quality as a management tool”, New Directions for Program Evaluation, pp. 113-126.
} 
de capacidades de los beneficiarios. Parte integral de AC ha sido, desde su creación, la realización de evaluaciones externas con objeto de conocer el desempeño e impacto de los subprogramas y proyectos que la conforman, así como identificar los factores que obstaculizan su instrumentación para el establecimiento de un proceso de mejora continua en todas y cada una de sus etapas. La evaluación del Programa abarca tres funciones primordiales: la administrativa; la política (transparencia en el ejercicio del gasto público y la rendición de cuentas); y, finalmente, la de gestión, que señala la importancia de la evaluación como herramienta de la planeación para futuros ejercicios.

Acorde con el compromiso adquirido en el Plan Nacional de Desarrollo 2001-2006, el Gobierno Federal ha evaluado año con año a AC en los diferentes estados de la República. Para ello, y a través de un acuerdo con el Gobierno Federal, la Organización de las Naciones Unidas para la Agricultura y la Alimentación (FAO) apoyó la realización de evaluaciones por Entidades Evaluadoras Estatales (EEE), contratadas para este efecto por los Comités Técnicos Estatales de Evaluación (CTEE), responsables de la calidad y del contenido de los informes finales. Las evaluaciones se desarrollaron según lo establecido en el Esquema Organizativo para la evaluación de los Programas de Alianza para el Campo, bajo la conducción de la Coordinación General de Enlace y Operación ${ }^{14}$. En este contexto, el apoyo de la FAO consistió básicamente en el desarrollo de una metodología de evaluación, en el soporte técnico y metodológico continuo a las EEE y a los CTEE durante el proceso de evaluación estatal, así como el desarrollo y gestión del sistema informático con el que se integraron los resultados estatales y en el ámbito nacional. En su operativa cada CTEE se ha hecho responsable de contratar a las EEE, conducir el proceso de evaluación estatal y de revisar y calificar los informes de evaluación elaborados.

Según la normativa, la utilidad fundamental de la evaluación de los programas de Alianza es brindar información objetiva y elementos de juicio para que los funcionarios, en sus diferentes niveles, puedan realizar su quehacer de manera más fundamentada y su actuación sea más efectiva en la solución de los grandes problemas hoy presentes en el agro mexicano (no sólo satisfacer la obligación administrativa establecida mediante normas), en tanto que para la FAO la evaluación de AC debe volverse en el país una costumbre que fortalezca las instituciones que participan en el desarrollo agropecuario nacional.

Programa de Fomento Agrícola en Yucatán. Durante el período 1996-2001 el rubro más apoyado en el estado fue la implementación de proyectos de riego tecnifi-

\footnotetext{
${ }^{14}$ SAGARPA-Gobierno del Estado de Yucatán (2006), "Programa de Fomento Agrícola. Informe de evaluación estatal 2005”, Yucatán. 
Aspectos relevantes del diseño, gestión e impactos del programa

de Fomento Agrícola en el estado de Yucatán 2002-2005

cado y de manejo del suelo y agua para impulsar la rentabilidad de este subsector. FA en esta primera etapa dispuso de los recursos para determinados componentes sin conexión entre sí (Ferti-irrigación, Mecanización, Kilo por Kilo y Fomento Citrícola), que eran de mayor alcance en cuanto al número de productores beneficiados. El Programa otorgó apoyos en diferentes ámbitos de la actividad agrícola (según necesidades identificadas); pero la falta de un enfoque sistémico provocó que estos generaran resultados que no se observaron en las estadísticas estatales. El productor se benefició en lo particular, no así los niveles de producción e ingreso del subsector $^{15}$. Por ello, y con el fin de reorientar FA hacia nuevos objetivos, se modificó en 2002 el paquete de apoyos que lo conformaban, integrándolos en tres subprogramas: inversión y capitalización, investigación y transferencia de tecnología e integración y fortalecimiento de cadenas agroalimentarias.

Por lo anterior, a partir de 2002, se denotaron cambios en el direccionamiento de los apoyos, destacándose la asignación de recursos para la investigación y transferencia de tecnología agrícola y, a partir del año 2004, para el fortalecimiento de los Sistemas Producto, esto una vez definidas las cadenas de atención prioritaria (6.4 \% en 2004 y 3.9 \% en 2005 del total del presupuesto ejercido). La asignación presupuestal destinada a la integración y fortalecimiento de estos sistemas fue pequeña, componente básico para la venta o compra de insumos y servicios, ya que la falta de capacidad de negociación del productor en estos mercados requiere de mejores condiciones para sus transacciones. La parte del presupuesto asignado a investigación y transferencia tecnológica ${ }^{16}$ fue, en promedio, de un tercio del total (45.5\% en 2002, $42.2 \%$ en 2003, $29.2 \%$ en 2004 y $30 \%$ en 2005); podría considerarse a ésta como alta, sin embargo se constata en las estadísticas el poco impacto que tuvo sobre los niveles de rendimiento en los principales cultivos.

El ejercicio financiero de FA en el período 2002-2005, en términos generales, mostró crecimiento, aunque no alcanzó el nivel de los ejercicios precedentes (en 1996 fue de 112,634.9 miles de pesos contra 40,359.9 miles de pesos en 2005). Los recursos destinados al fomento de la inversión y capitalización, en particular, resultaron de mayor magnitud con el 58.9 \% del total del Programa en ese período, y se incrementaron a partir de 2003; le siguió en importancia el de Investigación y Transferencia de tecnología (37.9 \%). Por el monto ejercido de 2001 a 2004, el rubro de mayor importancia lo constituyó el de sistemas de riego con el 48.8 \% del total operado, esto con el fin de impulsar la rentabilidad del subsector; en tanto los apoyos para la

${ }^{15}$ SAGARPA-Gobierno del Estado de Yucatán (2005), "Programa de Fomento Agrícola. Informe de evaluación estatal 2004”, Yucatán.

${ }^{16}$ En este subprograma, un 20 \% de los recursos se destinaron a la transferencia, lo cual resultó bajo; el productor al beneficiarse de otros componentes, necesita capacitación para su correcto manejo o aplicación. 
prevención del virus de la tristeza de los cítricos (VTC) ocupó el segundo sitio con el 29.6 \%. La estructura del presupuesto programático del año 2005 mostró también prioridad para con el subprograma de inversión y capitalización, al cual se le asignó aproximadamente el 66 \% del total, y de sus componentes se apoyó principalmente a la adquisición de sistemas de riego $(28.9 \%)^{17}$.

En lo que respecta al número y tipo de beneficiarios del Programa, en el quinquenio 2001-2005 se benefició a un total acumulado de 9, 462 productores, pero debido a que en 2002 se canceló el Programa de Kilo por Kilo, que incentivaba el uso de semillas mejoradas (benefició a 4,447 productores en 2001), el número de productores registró un notable descenso, pues en 2005 fue de sólo 740. Del total de beneficiarios en el periodo, el $92 \%$ estuvo conformado por productores de bajos ingresos clasificados en el estrato de en transición (Tipo III) ${ }^{18}$. Del total de éstos, el $42 \%$ recibieron apoyos por medio del Programa Kilo por Kilo y el 18 \% los recibió del programa citrícola, orientado a la prevención del VTC; ambos programas estuvieron vigentes hasta el año 2001 y fueron ejemplo de la entrega masiva de apoyos, característica por la que sus impactos fueron poco claros. El tercer componente de importancia fue el de fomento productivo y reconversión productiva, cuyo número de beneficiaros represento el $15.8 \%$ del total acumulado, este último componente es de importancia para el estado, ya que por medio de él actualmente se canalizan las plántulas o injertos resistentes al VTC ${ }^{19}$.

En lo que se refiere al valor real de las inversiones federales y estatales ejercidas en FA durante el período 2001-2005, se observa que se incrementó significativamente y su tasa fue de $42.9 \%$, como se indica en el cuadro 1 .

Cuadro 1

Recursos ejercidos a valores reales en el Programa de Fomento Agrícola en el período 2001-2005

\begin{tabular}{|c|c|c|c|c|c|c|c|}
\hline \multirow{2}{*}{$\begin{array}{c}\text { Ejercicio } \\
\text { Presup. }\end{array}$} & \multicolumn{6}{|c|}{ Aportaciones al Programa (Miles de pesos) } & \multirow{2}{*}{$\begin{array}{c}\text { Total } \\
\text { acumulado }\end{array}$} \\
\hline & Federal & Acum. & Estatal & Acum. & Productor & Acum. & \\
\hline 2001 & $18,174.7$ & $18,174.7$ & $2,405.3$ & $2,405.3$ & 879.5 & 879.5 & $21,459.6$ \\
\hline 2002 & $17,704.1$ & $35,031.2$ & $7,643.0$ & $9,936.1$ & $18,048.9$ & $18,887.4$ & $63,854.8$ \\
\hline 2003 & $21,232.1$ & $54,601.0$ & $6,291.0$ & $15,755.7$ & $24,689.8$ & $42,681.0$ & $113,037.7$ \\
\hline 2004 & $31,671.6$ & $80,292.7$ & $4,261.8$ & $18,292.0$ & $22,223.6$ & $60,230.2$ & $158,814.9$ \\
\hline 2005 & $26,119.6$ & $99,459.2$ & $3,292.8$ & $20,000.7$ & $10,947.5$ & $65,961.9$ & $185,421.8$ \\
\hline Totales & $99,459.2$ & & $20,000.7$ & & $65,961.5$ & & \\
\hline Part. (\%) & 53.6 & & 10.8 & & 35.6 & & 100.0 \\
\hline
\end{tabular}

Fuente: Informe de evaluación estatal. Programa de Fomento Agrícola. Yucatán. 2006.

Nota: Valores nominales deflactados con el índice Nacional de Precios Productor, 2002=100

\footnotetext{
${ }^{17}$ SAGARPA-Gobierno del Estado de Yucatán (2006), op. cit. p. 22.

${ }^{18}$ Estos productores, a diferencia de los de zonas marginadas o no marginadas, poseen un mayor nivel de recursos económicos y mejores medios de producción, características que los hacen candidatos idóneos para los subprogramas de FA y en especial el de fomento a la inversión y capitalización.

${ }^{19}$ SAGARPA-Gobierno del Estado de Yucatán (2006), op. cit. p. 23.
} 
Aspectos relevantes del diseño, gestión e impactos del programa

de Fomento Agrícola en el estado de Yucatán 2002-2005

Los recursos ejercidos en el Programa fueron en su mayor parte contribuciones del gobierno federal (53.6 \%); el gobierno estatal participó con el 10.8 \%. Por su parte, las aportaciones de los productores sumaron aproximadamente el $35.6 \%$ del total acumulado de inversión en FA (185.4 millones de pesos constantes). Se aprecia que la contribución anual de los gobiernos federal y estatal fue variable; por ejemplo, de una contribución estatal de 30.2 \% en el año de 2002 pasó a 11.2 \% en 2005.

Efectos del Programa y atención a la problemática agrícola. La contribución del ingreso agrícola en el PIB del estado de Yucatán fue en promedio de $1.13 \%$ del año 2000 al 2004 e indica que la producción tuvo una contribución marginal en el valor total generado; pero su tendencia fue ascendente y pasó de $1.02 \%$ en 2000 a 1.24 \% en 2004, lo que refleja en alguna medida el esfuerzo emprendido en el campo por el gobierno, productores y otros involucrados en FA. En lo particular, el ingreso nominal agregado de los productores beneficiados por el Programa experimentó un incremento promedio de $56 \%$, el cual influyó principalmente el aumento en la superficie cosechada (55.4\%); pero si se considera la variación de $4.62 \%$ en el índice general de inflación ocurrida en el estado para el mismo período, el precio agregado real se redujo en 4.5 puntos porcentuales, lo cual evidencia una disminución del poder de compra del precio pagado al productor.

De los principales cultivos en el estado, maíz (174,805 ha), henequén (68,087 ha), cítricos (21,137 ha), frijol (4,373 ha) y papaya maradol (1,026 ha), el grupo de subprogramas de FA destinó mayores apoyos al primero y tercer cultivos. Sin embargo, no se consideró la necesidad de adaptar los planes impulsados por el estado a la situación económica, productiva y cultural de los beneficiarios, de tal forma que aunque el apoyo se otorgó mediante la entrega de maquinaria y equipo o infraestructura -como los invernaderos- el productor no pudo (y muchas veces no quiso) obtener un beneficio de lo que le fue otorgado porque no dispuso de la técnica para su uso o porque no valoró lo que recibió. Los apoyos de FA (aunque contribuyeron a paliar la pobreza campesina) no fueron suficientes para impulsar el fortalecimiento de la competitividad de las cadenas agroalimentarias y la diversificación de fuentes de empleo, por lo que estuvo lejos de impulsar el fortalecimiento de este objetivo. Por ejemplo, el índice de productividad agregada de los cultivos apoyados fue en promedio de 1.002 en el año 2005, lo cual evidencia que antes y después del apoyo no se registró un incremento significativo en los rendimientos, situación que se explica principalmente por los tipos de inversiones que se realizaron; el apoyo se orientó a la sustitución de plantas de cítricos o de equipos de riego (rodado por aspersión o micro aspersión), lo cual no incidió sobre la productividad (el área de invernaderos es pequeña). En lo que respecta al empleo, se obtuvo que se requieren cuatro agricultores beneficiados por el Programa para generar un empleo contratado y 34.8 de ellos para generar un 
Miguel Ángel Magaña Magaña y Carlos Enrique Leyva Morales

empleo familiar; diferencia que obedeció al hecho de que en las explotaciones pequeñas (menos de una hectárea) el propietario es quien realiza la mayor parte de las labores de cultivo ${ }^{20}$.

Por otra parte, los productores de los cultivos más beneficiados (maíz y cítricos) siguieron enfrentando los mismos problemas que no han permitido que su actividad se desarrolle plenamente, entre los que se encuentran el deficiente manejo de las plantaciones, falta de asistencia técnica, falta de mecanismos para el control integral de plagas y enfermedades y precios bajos. Esta situación limitó fuertemente las posibilidades de inversión y mantuvo marginada la actividad maicera y citrícola de las nuevas tecnologías productivas (la principal reconversión productiva en el estado se ha dado por impacto del Programa en la sustitución de las plantaciones de cítricos susceptibles al VTC); cabe señalar que la naranja no entró al TLC, lo que limitó la posibilidad de su exportación.

En cuanto a la correspondencia entre la problemática del subsector agrícola de la entidad y la implementación del Programa y sus componentes, se tiene en primer término que el diseño de FA no consideró algún tipo de priorización por actividad o grupo específico de productores por apoyar, que permitiera al estado maximizar los posibles impactos de los apoyos en el subsector agrícola. Por consiguiente, el Programa respondió parcialmente a las necesidades y problemática observadas en el subsector (carencia de infraestructura, presencia de plagas y enfermedades, desvinculación de eslabones de la cadena productiva, entre otros) y, toda vez que no se contó con una planeación estratégica, limitó su operación a la atención de solicitudes conforme a su orden de llegada. Por otro lado, el monto total de sus recursos fue reducido en comparación con los otros programas que se desarrollan en la entidad (la participación de FA, considerando los montos reales pagados de 2001 a 2005, fue en promedio de 18.9 \% del total de recursos superado únicamente por el programa de Desarrollo Rural con $46 \%$ ), pues existieron diferencias entre los montos de inversión programados y los realmente ejercidos (el presupuesto 2005 para el Programa era originalmente de 35,295.8 millones de pesos, el cual se incrementó por reprogramación a 38,029.8 millones, un $7.7 \%$ más), además de que fueron menos sus beneficiarios (en 2005 $82.3 \%$ de los productores solicitantes fueron beneficiarios contra $88.3 \%$ en $2003^{21}$ ). Un tercer aspecto relevante fue la limitada relación de complementariedad entre FA y otros programas gubernamentales (en particular la vinculación entre FA y el Programa de desarrollo de capacidades en el medio rural), situación que no ha facilitado el desarrollo de las actividades productivas de alto impacto en el estado debido a que

\footnotetext{
${ }^{20}$ SAGARPA-Gobierno del Estado de Yucatán (2006), op. cit. p. 62.

${ }^{21}$ SAGARPA-Gobierno del Estado de Yucatán (2006), op. cit. p. 44. 
Aspectos relevantes del diseño, gestión e impactos del programa

de Fomento Agrícola en el estado de Yucatán 2002-2005

éstas requieren elevados niveles de inversión, servicios de asesoría y conocimientos técnicos especializados por parte de los productores.

Análisis del proceso de evaluación del Programa de Fomento Agrícola en Yucatán. Se puede decir que el tipo de evaluación de políticas que se ha utilizado en FA en el estado de Yucatán corresponde a la clasificación por objetivos según contenido de la evaluación; mide el diseño, la gestión y los resultados e impactos del Programa (con carácter ex-post).

Del análisis de las bases normativas de la evaluación realizada a FA, se encontró lo siguiente ${ }^{22}$ :

1.- La normativa fue elaborada por FAO-SAGARPA y expresada en la Guía Metodológica ya referida. Se ha coordinado a través de un Comité Técnico Estatal de Evaluación que publica las convocatorias para que las instituciones interesadas en participar en el proceso evaluatorio hagan sus propuestas y concursen en la asignación de los recursos para tal fin.

2.- Esta evaluación se ha realizado de forma anual con relación a los resultados del año anterior y se ha mantenido de forma continua desde 2002 en la entidad.

3.- Los indicadores medidos, derivados de las bases normativas para la evaluación del mismo, reflejan en gran medida los objetivos y resultados que se esperaban de la operativa y aplicación del Programa en el estado (resultado de los modelos evaluatorios por objetivos y descriptivos en que se ha basado el proceso), pero existen problemas en términos del seguimiento de éstos debido a que les falta reflejar la proporción de productores que aprovechan o adoptan en sus procesos productivos los beneficios y la tecnología que se les transfiere, además de que no se evalúan los resultados reales que en verdad interesan, muchas veces no contemplados (propio de los modelos analíticos y de los basados en los resultados de la evaluación); por ejemplo, la evaluación del factor político, que influye en la implementación de este programa gubernamental

De acuerdo con lo anterior, en la evaluación de Programa no se están contemplando todos los enfoques, los factores y las atenuantes de por qué no está funcionando el Programa (falta objetividad), no obstante los señalamientos de funcionarios de experiencia sobre el papel y la importancia de estos aspectos, los cuales no son considerados ni son motivo de crítica o de cambio alguno en el rediseño de la normativa, la implementación y la evaluación de FA (principalmente en materia de evaluar su eficiencia).

${ }^{22}$ Elaborado por los autores con los resultados de las entrevistas a informantes clave. 
En cuanto a los principales aciertos y deficiencias que presentó FA en el estado durante el período 2002-2005, de acuerdo con lo que marca la teoría de la evaluación de políticas públicas y desde la perspectiva de su diseño, gestión, seguimiento e impactos fueron:

En lo que respecta a aciertos, en materia de diseño, el primero fue su creación como Programa en el año 2002, al conjugarse parte de los programas que fueron creados en 1996 o posteriormente. FA se desarrollo transversal a las actividades económicas del estado y posteriormente integró el esquema de cadenas productivas para delinear las más importantes en la entidad (horticultura, chile habanero, cítricos, ornamentales y sábila, de las cuales funcionan adecuadamente las de cítricos y chile habanero). Otro acierto lo constituyó la investigación en materia agrícola que se apoyó en el Programa y que se derivó de las demandas que realizaron los productores hacia los centros correspondientes. En tanto que el tercer acierto fue la atención de los problemas del agua en la región, cuya finalidad fue para buscar el mejor uso de este recurso y su sustentabilidad.

En materia de gestión se constató que:

1. Los recursos del Programa FA han sido otorgados conforme la localización geográfica de los cultivos en el estado; por tanto se han atendido las áreas productoras, principalmente la zona sur, que es la de mayor relevancia agrícola, y la zona exhenequenera.

2. El Programa ha servido de puente entre las instituciones de investigación, información y tecnología que generan, y los productores como demandantes.

3. El productor conoce como acceder al Programa y los requisitos que tiene que cubrir; se han invertido recursos en el campo bajo una normativa ya conocida, aunque los apoyos no se están otorgando en igualdad de oportunidades para todos ellos.

4. El gobierno del estado, en la parte que le corresponde de la operativa de FA, ha ido de menos a más en su eficiencia al respecto; subió su calificación de tres a siete puntos según los parámetros de evaluación de FAO, limitado sólo por el efecto de la política gubernamental, la dispersión de recursos y la falta de planeacion en la aplicación de éstos.

5. La normativa se ha hecho cada vez más sencilla, de tal manera que el estado tiene hoy en día mayores atribuciones para aportar y distribuir las acciones y recursos a su manera; las bases normativas quedan sólo como lineamiento general por seguir, se han adecuado un poco los requerimientos del subsector agrícola estatal y han sido flexibles para que éste retome la parte que le corresponde en cuanto a diseño y planeación. 
Aspectos relevantes del diseño, gestión e impactos del programa

de Fomento Agrícola en el estado de Yucatán 2002-2005

Respecto al seguimiento del Programa, los aciertos de FA se encuentran principalmente en que este proceso se lleva a cabo en el Programa, esto en el reporte de los logros alcanzados en términos de las metas programadas y en el control que se tiene de los productores beneficiados por el Programa, y en la evaluación externa de FA, lo cual resulta una ventaja por el ejercicio completo de los recursos destinados a la realización del mismo.

En cuanto a materia de impactos, entre sus aciertos se pueden considerar que la eficacia de FA se ha logrado en algunos aspectos, dado que se cumplen con los indicadores de las acciones físicas e inversiones realizadas, pero bajo la base normativa establecida (por ejemplo, en materia de adopción de la tecnología que se genera en las instituciones de investigación relacionadas con el subsector ésta ha sido del 80 \%), que se cumplió con las metas y las entregas de los beneficios del Programa a la población objetivo (físicos y financieros) y que año con año se han venido mejorando los indicadores de eficacia de la implementación del Programa en la entidad.

En lo que concierne a las deficiencias de FA, en materia de diseño se observó que no obstante las atribuciones que le dio la federalización al gobierno estatal, donde el estado debió de retomar su propio diseño bajo un esquema de planeacion estratégica, éste no ha estado presente y no hay documentos que lo avalen; por lo tanto, no se ha realizado un diseño adecuado del Programa para resolver la problemática del subsector agrícola. Los tiempos constituyen otro factor que no permite hacer un diseño a futuro de carácter multianual de FA; otra falla es la falta de una identificación real de la problemática agrícola del estado que oriente objetivamente el enfoque de los planes y programas; también existe dificultad en cuanto a los mecanismos de transferencia de los resultados de investigación en términos de que en el plan no se especifica quién va ser el encargado de realizar ésta hacia los productores del campo; una cuarta deficiencia lo constituye el hecho de que el Programa, a pesar de que se ha regionalizado, no aplica en todos los rubros de apoyo (por ejemplo, la compra de maquinaria -tractores- y equipo en algunos casos no ha correspondido a las características de la zona); por último, en cuanto al diseño de los subprogramas, se observó que en éstos no se tiene en cuenta las metas planteadas en el Plan Estatal de Desarrollo (PED), se ha avanzado más en cuanto a las oportunidades que se ofrecen que en relación a las solicitudes de apoyos, además de que lo que se planea no necesariamente se está haciendo (resultado de la entrevista). Por todo lo anterior, y considerando los lineamientos que la teoría relativa a la evaluación de políticas públicas señala, se puede afirmar que FA ha sido un programa limitado y con impacto parcial en el estado en virtud de que si bien es coherente, es decir, presenta una óptima correlación entre las actuaciones que realiza, sus medios y sus objetivos, no es racional en el sentido de que exista relación entre los problemas que atiende, sus causas y sus objetivos. 
En cuanto a la gestión de FA, las principales deficiencias se encuentran en que en el Estado no hay un esquema de caracterización real o tipología de los productores del sector rural, por lo que no se ha podido diferenciar niveles y establecer a cuáles les corresponde recibir los apoyos del Programa, ya que en la práctica los principales criterios para la asignación se han regido por las directrices centralizadas, la disponibilidad de recursos recibidos y la definición de los componentes hacia los que se destinarán los apoyos. También en la dificultad que existe en la transferencia de tecnología por la falta de coordinación y comunicación entre las instituciones que participan en el subsector; otra deficiencia es que el desarrollo del Programa no se apega a la planeación establecida en el PED, no obstante de que esta gestión recae casi en el $100 \%$ en el gobierno del estado (SDRyP y sus diferentes direcciones) y que a la SAGARPA sólo le corresponde fungir como guía normativa; otra mas es que en el desempeño de los funcionarios relacionados con la gestión de FA, al estar inmersos en reuniones de comités o en arreglos institucionales para consolidar la organización de las instancias necesarias, no pueden ser eficientes a este respecto, más objetivos y tampoco revisar a profundidad la evaluación; por último, con relación a las líneas establecidas para apoyar en la parte operativa, en que los productores reciben los beneficios del Programa más bien por cuestiones de tipo político (identificación con el grupo en el poder), esto ocurre en forma velada. Considerando las citadas deficiencias, el enfoque teórico bajo el cual se ha desarrollado la evaluación de la gestión de FA, se puede decir que ha sido el de tipo integral de gestión del ciclo de vida del proyecto, el cual comprende las fases de programación de las actuaciones, de diseño de los mecanismos de ejecución y gestión del Programa y la de evaluación por objetivos. Sin embargo, otras variables relacionadas con la capacidad de los organismos implicados en la gestión de FA no han sido considerados, siendo algunas de éstas la capacidad de definir la estrategia de acción y la disponibilidad de las adecuadas y precisas estructuras para dirigir las complejas actividades internas de FA y que la estructura de la organización responsable (SDRyP) esté en consonancia con los recursos humanos, cultura organizativa y proceso de dirección adecuada.

En materia de seguimiento, las deficiencias se encuentran en la operativa real de FA, ya que ésta no se realiza como debe ser por cuestiones del presupuesto, falta de personal, etc. Falta también que se dé esta acción a nivel de campo (se hace normativamente), dado que existe un problema de desfasamiento en la operación, en los avances, en las acciones y en todo el proceso ante la falta de una planeación adecuada; fuerte influencia tiene en esto los instrumentos de acopio de información utilizados (la cédula y la guía de entrevista), los cuales no miden si se produjeron cambios en el proceso de producción y organización del agricultor, si se gasto más o menos por el Programa, así como tampoco proporcionan indicadores que permitan calcular los avances en materia de eficiencia; la metodología no se ha fijado en el impacto económico y 
Aspectos relevantes del diseño, gestión e impactos del programa

de Fomento Agrícola en el estado de Yucatán 2002-2005

sí en el social y en indicadores de las inversiones, pero éstos no resultan confiables (no incluyen reactivos de prueba que indiquen el avance o retraso del beneficiario por haber incorporado cambios). Por último, no existe un sistema de información único que haga al Programa más transparente, ya que cada quien lo maneja como le es más adecuado; el gobierno del Estado no apoyó el establecimiento del sistema de información denominado SISER porque no se adecua a sus condiciones y forma de trabajo; sin embargo, el sistema que ellos diseñaron para tal efecto tampoco lo utilizaron. Se puede afirmar por consiguiente que FA no está alcanzando en forma adecuada el objetivo de ser un sistema de seguimiento, es decir, la verificación periódica de lo que se está realizando desde la perspectiva física y financiera. Por tanto, su sistema de evaluación no facilita la toma de decisiones sobre las acciones correctivas a emprender en el Programa, y a la organización responsable de su aplicación no le facilita el cumplimiento de sus objetivos, ni la medición de los progresos realizados para la consecución de éstos.

Por último, en materia de impactos, las deficiencias de FA se relacionan en primer lugar con las limitaciones económicas de los productores para acceder a la tecnología generada; en segundo lugar, no se cubre con los parámetros o indicadores medidos en un $100 \%$ y en tercero no se cumple en tiempo con las metas y las entregas de los beneficios a la población objetivo. El apoyo no es entregado con oportunidad en la mayoría de los casos y existe un cuello de botella en la operación, por lo que resulta tedioso y burocrático el proceso. Por otra parte, es importante mencionar que en la evaluación de la eficacia de FA se han utilizado como técnicas principales presupuestos, estadísticas, encuestas y entrevistas, dejando de lado otras más efectivas que la literatura recomienda, como la observación directa, visitas in-situ o la técnica Delphi, sobre todo por los elementos cualitativos inmersos.

En cuanto a la eficiencia de FA en la entidad, se puede decir que ésta, en opinión de los responsables de su seguimiento, se ha logrado en forma relativa debido entre otras causas a la falta de coordinación entre las dependencias relacionadas con el subsector para integrar y enlazar los apoyos que existen, a la falta de continuidad en la implementación y manejo del Programa porque cada vez que hay cambios en la administración federal o estatal se inicia de cero prácticamente, perdiéndose la capacitación del personal en todos sus niveles operativos, y a que, tomando en cuenta el costo de la estructura del Programa, éste no ha resultado tampoco eficiente, no por culpa sólo del operador del Programa en el Estado ${ }^{23}$, sino también con relación a la población objetivo y los recursos que debieran aportar una u otra parte. Hay que señalar que si

\footnotetext{
${ }^{23}$ Existen variables que están fuera de control de los operadores, como la disponibilidad de los recursos federales que definen la apertura de ventanillas de atención.
} 
Miguel Ángel Magaña Magaña y Carlos Enrique Leyva Morales

bien se esboza la medición de eficiencia en FA desde su inicio, en la operativa de las EEE no se está realizando ésta, ni tampoco se considera alguna técnica en particular para ello, lo cual se evidencia en los reportes de evaluación del mismo.

Con base en lo expuesto, si bien los reportes de evaluación de FA indican que en términos de eficacia su orientación e importancia como medio de apoyo ha sido congruente con el propósito de mejorar las condiciones en las que se desarrollan las actividades agrícolas en el estado y, asimismo, reconocen que a pesar de la limitada disponibilidad de recursos se ha estado avanzando, pero a ritmo lento, comparado con el de las necesidades que imponen los cambios socioeconómicos que ocurren en el medio rural de Yucatán y en los mercados. Se puede afirmar que la evaluación del Programa FA no está permitiendo determinar y cuantificar en forma objetiva y completa los cambios en términos de eficiencia, efectos estructurales y sinergias que se han producido en la población objetivo debido a que en la práctica de ésta no se han medido ni separado los efectos indirectos provocados por el Programa, como el grado de satisfacción de la población objetivo, el cambio de las expectativas y conductas de los participantes, el análisis conjunto entre los programas (federales y estatales) orientados al subsector, entre otros, y a que en el proceso de valoración de FA no se está considerando la existencia de valores y ponderaciones, la mayor parte de las veces no explícitos y cargados de juicios de valor (políticos y sociales), en la elección y aplicación de las técnicas para evaluar eficacia y eficiencia; tampoco se está tomando en cuenta la evolución que la evaluación de impactos de políticas ha tenido hacia un concepto más amplio, donde los aspectos cualitativos cobran cada vez mayor relevancia, principalmente porque sus métodos aportan dinamismo y variedad de perspectivas (el Análisis Costo-Beneficio, el Análisis de Frontera y la metaevaluación).

\section{Conclusiones}

1. En materia económica, y con relación a la capitalización de las unidades de producción agrícola del estado, el Programa de Fomento Agrícola ha incidido en la mejora técnica de las unidades de producción de los agricultores beneficiados, lo cual influye en menores costos, medios de producción e incrementa la utilidad en el año de recepción del apoyo. Asimismo, el Programa le apostó al desarrollo del cultivo de cítricos, y el área donde se aprecia el mayor impacto fue el cambio de variedades por las tolerantes al VTC. No obstante lo anterior, en lo general se considera que estos resultados han sido pobres, principalmente por la atomización del apoyo otorgado, la disminución del poder de compra de los productores y el bajo número de beneficiados (el Programa ha servido más como apoyo social a los productores, obedeciendo a condiciones políticas y a los requerimientos de la población rural). 
Aspectos relevantes del diseño, gestión e impactos del programa

de Fomento Agrícola en el estado de Yucatán 2002-2005

2. En materia social, el Programa ha apoyado a grupos de productores y a la población rural (principalmente en proyectos de horticultura y citricultura); ha reducido, aunque en un bajo porcentaje, la migración al generar fuentes de empleo temporal; y ha promovido la participación de los productores en el proceso de reconversión productiva.

3. Respecto al diagnóstico de la evaluación del diseño, gestión e impactos del Programa de Fomento Agrícola, se concluye que ésta no fue factor determinante en el crecimiento observado en el volumen y valor de la producción, el empleo y el ingreso bruto de los productores que integran este subsector en Yucatán de 2002 al 2005 debido a lo siguiente:

a) La evaluación realizada al Programa carece de objetividad, pues ésta se centra principalmente en los referentes estadísticos disponibles (según la normativa) y no se consideran los efectos indirectos derivados de FA (con el fin de tener indicadores más reales), así como tampoco se están empleando por la EEE las técnicas e instrumentos que la literatura recomienda (producto de la evolución teórica) para la medición de eficiencia, efectos estructurales y sinergias (el elemento político constituye un factor determinante en este aspecto), ni se está analizando el estado del sistema de evaluación.

b) El hecho de que en la operativa del Programa falta continuidad en los responsables de la gestión de FA, de que el costo de su estructura no es eficiente, de que el proceso de evaluación no ha logrado permear adecuadamente en el quehacer de los operadores (creen que se les está auditando) y de que en la aplicación del Programa, posterior a las evaluaciones realizadas por las EEE, no se están considerando las observaciones y recomendaciones hechas en éstas por funcionarios y productores.

c) Para la mayoría de los funcionarios de gobierno la evaluación realizada se considera sólo como un instrumento que se tiene que cumplir o realizar para mejorar la ejecución del Programa (medio de control) y, en consecuencia, no se está cumpliendo con el objetivo más amplio de este proceso (en cuanto a seguimiento) que es racionalizar la toma de decisiones, formar a los participantes, generar información y reformar las practicas administrativas. No se aprovecha su valor dado el alcance limitado de la metodología utilizada (análisis presupuestal, estadísticas agrícolas, encuesta a productores y entrevista a funcionarios).

d) Los instrumentos de acopio de información utilizados en la evaluación (cédula y guía de entrevista) no realizan el seguimiento de FA, no miden los efectos indirectos ni los cambios técnico-económicos en el agricultor, ni proporcionan indicadores que permitan calcular si hubo avance en materia de eficiencia. 
4. Por último, se recomienda, para corregir las deficiencias observadas, que se mejoren los resultados económicos de FA -principalmente la racionalidad en la asignación de recursos- el número de productores beneficiados y los indicadores de impacto de primer nivel (ingreso y empleo); en lo que respecta a la evaluación del Programa, para que cumpla con sus objetivos hacia el productor, que al interior se incluyan en los instrumentos de acopio de información reactivos que brinden indicadores de tendencia de la producción de parcelas, rendimientos, costos, beneficios económicos entre otros, que el personal técnico que acopia la información sea homogéneo en conocimientos y capacitación, que la entrevista se realice en la unidad de producción, no en el domicilio del productor y principalmente, que se realice la evaluación de la eficiencia de FA por la EEE (mayores recursos y desarrollo de capacidades) a fin de contar con la confiabilidad y validez que sería deseable; al exterior, que se analice el estado del sistema de evaluación, o metaevaluación, insumo imprescindible para planear estratégicamente su desarrollo futuro.

\section{Bibliografía}

BRIK, A. S. (1983), "Stakeholder-based evaluation”, New Directions for program evaluation, No 17, San Francisco: Jossey-Bass, pp. 3-14.

Cardozo, Myriam (2005), "La evaluación de políticas y programas sociales en México: 1998-2004", Tesis para obtener el grado de Doctor en Ciencias Sociales, UNAM, México.

Chelimsky, E. (1983), "The definition and measurement of evaluation quality as a management tool”, New Directions for Program Evaluation, № 18, pp. 113-126.

Cohen, Ernesto (2001), Los desafíos de la reforma del Estado en los programas sociales: tres estudios de caso, Santiago de Chile, CEPAL (Serie Políticas Sociales, $\mathrm{N}^{\mathrm{o}} 45$ ).

García Valderrama, Teresa y Yolanda Calzado Cejas (1996), "Metodología de evaluación de la eficiencia en las entidades públicas”, reproducido de Presupuesto y Gasto Público, No 18, pp. 189-202.

Hernández Sampieri, R. C. Fernández Collado y P. Baptista Lucio (1991), Metodología de la investigación, México: McGraw Hill.

Joint Committee on Standards for Educ. Evaluation (1988), The personnel evaluation standards: How to assess systems for evaluating educators, Newbury Park, CA: Sage. 
Aspectos relevantes del diseño, gestión e impactos del programa

de Fomento Agrícola en el estado de Yucatán 2002-2005

Osuna, J. y C. MárQuez (2000), Guía para la evaluación de políticas públicas, Instituto de desarrollo regional, España

Rebolloso, E., B. Fernández-Ramírez., P. Cantón, Y C. Pozo (2000), El papel de la investigación cualitativa en la evaluación de los servicios universitarios, Cuadernos IRC, $\mathrm{N}^{\circ} 4$, pp. 65-82.

(2002), Metaevaluation of a total quality management evaluation system, Psychology in Spain, Vol. 6, № 1, pp.12-25.

Sagarpa-Gobierno del estado de Yucatán (2005), Programa de Fomento Agrícola. Informe de evaluación estatal 2004, Yucatán.

SAgarpa-Gobierno del estado de Yucatán (2006), Programa de Fomento Agrícola. Informe de evaluación estatal 2005, Yucatán.

Scotт, John (2007), SDTE 395 Agricultural Policy and Rural Poverty in México, Documentos de trabajo, División Economía, CIDE.

SCRIVEn, M. (1969), An introduction to metaevaluation Educational Product Report, $\mathrm{N}^{\mathrm{o}}$ 2, pp. 36-38.

Stufflebeam, D.L. y A.J. Shrikfield (1987), Evaluación sistemática: guía teórica y práctica. Barcelona, Paidos.

Trujillo Félix, Juan de Dios (2004), Las reformas de las políticas agrícolas de Estados Unidos, la Unión Europea y México. Análisis comparativo e implicaciones para México, Tesis doctoral, CIESTAAM, Chapingo, Estado de México.

Wholey, J. S. (1983), “Evaluation and effective public management”, Boston: Littie Brown.

(A) 
